\title{
Resequencing genomes of the Russian native Baikal and Tuva sheep breeds
}

James Sweet-Jones

Royal Veterinary College, University

of London, UK
Nikolay Yudin

Kurchatov Genomics Center,

Institute of Cytology and Genetics, SB RAS

Novosibirsk, Russia

\author{
Denis M. Larkin \\ Royal Veterinary College, University \\ of London, UK \\ Institute of Cytology and Genetics, SB RAS \\ Novosibirsk, Russia
}

Twenty animals of the Baikal and Tuva native Russian sheep breeds have been resequenced resulting in $\sim 17$ million SNP identified, of which approximately $30 \%$ were absent from the SNPdb database at the NCBI. Analysis of the sequencing data has identified genome areas likely to be under selective pressure in these Siberian sheep populations. The Tuva breed showed a much larger number of areas with an increased fixation index (Fst) relative to the Baikal breed when we used as a contrast 14 sheep genomes from the UK. This may be due to the remote phylogenetic position of the Tuva breed from the European sheep. Interestingly, almost every region with an elevated fixation index in both breeds contained a protein-coding gene and about $50 \%$ of them contained nonsynonymous nucleotide changes with high Fst values, suggesting possible effect of these substitutions on the protein functions in these breeds. For the Tuva breed, the highest Fst was observed in the PPP1CC gene area, which was shown earlier to be involved in the process of fat storage in livestock. Another gene (HHIP), which affects the differentiation of adipocytes, is under likely selection pressure in the Tuva breed on chromosome 17. In the Baikal breed, the highest fixation index was observed in the area of the gene ZNF280B, the transcription factor that regulates many other genes. The second top significant area of the Baikal breed contained the RALY gene, which, as previously shown, affects the quality of wool in sheep. A number of other genes affect immunity (TARBP1 and CLSTN1) and may thus be associated with adaptation to local conditions. Since we were able to identify nucleotide variations under selective pressure in these two populations, these variants can be tested for effects in genomic selection programmes in Russian sheep breeds.

\section{Acknowledgments}

This work was funded by the RSF grant 19-76-20026. 